\title{
Clear-PEM: A PET imaging system dedicated to breast cancer diagnostics
}

M.C. Abreu ${ }^{\mathrm{a}}$, D. Aguiar ${ }^{\mathrm{f}}$, E. Albuquerque, F.G. Almeida ${ }^{\mathrm{f}}$, P. Almeida ${ }^{\mathrm{c}}$, P. Amaral ${ }^{\mathrm{a}}$,

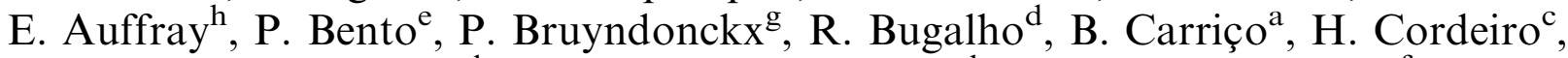
M. Ferreira ${ }^{\mathrm{a}}$, N.C. Ferreira ${ }^{\mathrm{d}}$, F. Gonçalves ${ }^{\mathrm{e}}$, P. Lecoq ${ }^{\mathrm{h}}$, C. Leong ${ }^{\mathrm{e}}$, F. Lopes ${ }^{\mathrm{f}}$, P. Lousã ${ }^{\mathrm{e}}$, J. Luyten ${ }^{\mathrm{a}}$, M.V. Martins ${ }^{\mathrm{c}}$, N. Matela ${ }^{\mathrm{c}}$, P.R. Mendes ${ }^{\mathrm{a}}$, R. Moura ${ }^{\mathrm{a}}$, J. Nobre ${ }^{\mathrm{e}}$, N. Oliveira ${ }^{\mathrm{c}}$, C. Ortigão ${ }^{a}$, L. Peralta ${ }^{\mathrm{a}}$, J. Rego ${ }^{\mathrm{e}}$, R. Ribeiro ${ }^{\mathrm{a}}$, P. Rodrigues ${ }^{\mathrm{a}}$, A.I. Santos ${ }^{\mathrm{b}}$, J.C. Silva ${ }^{\mathrm{a}}$, M.M. Silva ${ }^{\mathrm{e}}$, S. Tavernier ${ }^{\mathrm{g}}$, I.C. Teixeira ${ }^{\mathrm{e}}$, J.P. Teixeira ${ }^{\mathrm{e}}$, A. Trindade ${ }^{\mathrm{a}}$, J. Trummer ${ }^{\mathrm{h}}$, J. Varela ${ }^{\mathrm{a}, *}$

\author{
${ }^{\mathrm{a}}$ LIP, Lab. de Instrumentação e Física Exp. Partículas, Portugal \\ ${ }^{\mathrm{b}}$ Hospital Garcia de Orta, Almada, Portugal \\ ${ }^{c} I B E B$ - Inst. de Biofísica e Eng. Biomédica, Lisboa, Portugal \\ ${ }^{\mathrm{d}}$ IBILI - Inst. Biomédica de Investigação em Luz e Imagem, Facul. de Medicina, University Coimbra, Portugal \\ ${ }^{\mathrm{e}}$ INEGI Inst. Eng. Mecânica Gestão Industrial, Porto, Portugal \\ ${ }^{\mathrm{f}}$ INESC-ID and INOV, Lisboa, Portugal \\ ${ }^{\mathrm{g}} V U B$, Brussels, Belgium \\ ${ }^{\mathrm{h}} C E R N$, Geneve, Switzerland
}

Available online 7 November 2006

\begin{abstract}
The Clear-PEM scanner for positron emission mammography under development is described. The detector is based on pixelized LYSO crystals optically coupled to avalanche photodiodes and readout by a fast low-noise electronic system. A dedicated digital trigger (TGR) and data acquisition (DAQ) system is used for on-line selection of coincidence events with high efficiency, large bandwidth and small dead-time. A specialized gantry allows to perform exams of the breast and of the axilla. In this paper we present results of the measurement of detector modules that integrate the system under construction as well as the imaging performance estimated from Monte Carlo simulated data.
\end{abstract}

(C) 2006 Elsevier B.V. All rights reserved.

Keywords: Positron emission mammography

\section{Introduction}

Positron emission tomography (PET) has been proposed as a complementary technique to X-ray mammography in the diagnosis and treatment monitoring of breast cancer. In the recent years, a significant progress has been made in the development of compact systems, specially designed for breast imaging known as positron emission mammography (PEM) cameras $[1,2]$. Belonging to this new generation of

\footnotetext{
${ }^{*}$ Corresponding author. Tel.: +41227678 973; fax: +41227678940 .

E-mail address: joao.varela@cern.ch (J. Varela).
}

scanners is the Clear-PEM scanner under development by the PEM Consortium in the framework of the Crystal Clear Collaboration at CERN [3,4].

The detector is based on pixelized LYSO:Ce crystals optically coupled on both extremities to avalanche photodiodes (APD) and readout by a fast low-noise electronic system. A dedicated digital trigger and data acquisition system is used for on-line selection of coincidence events with high efficiency, large bandwidth and small dead-time. The scanner consists of two compact and planar detector heads with adequate dimensions for breast and axilla imaging. A dedicated gantry is being built to allow the 
rotation of the detector heads in breast exams as well as to permit exams of the axilla region. The Clear-PEM scanner is developed with three main guidelines: low random background; high sensitivity; and spatial resolution of the order of $2 \mathrm{~mm}$. The first requirement arises from the fact that the scanner must cope with a large single photon rate. In order to increase the sensitivity the Clear-PEM imaging system allows to exploit Compton interactions in the detector. Finally, in order to deliver the required spatial resolution allover the field-of-view (FoV) without compromising the sensitivity by restricting the angle of the accepted lines-of-response, the detector is able to measure the depth-of-interaction (DoI) of the incoming photons [4].

\section{The Clear-PEM scanner}

\subsection{Detector system}

The Clear-PEM scanner consists of two parallel detector heads covering a $16.2 \times 14.1 \mathrm{~cm}^{2}$ FoV. Each head holds 96 detector modules with a packing fraction of about $52 \%$. In total there are 12,288 electronics readout channels. Modules are composed of a $2 \times 2 \times 20 \mathrm{~mm}^{3}$ LYSO:Ce 32 crystal array optically coupled on each side to a 32-pixel Hamamatsu S8550-01 APD array. Twenty-four modules are grouped in a mechanical structure called supermodule with about $4 \times 14 \mathrm{~cm}^{2}$. Each detector head is formed by four supermodules side by side. The Clear-PEM scanner is mounted on a dedicated robotic gantry that controls the detector head positions for breast examinations. During the exam the patient lays in prone position with the breast hanging through an aperture in an imaging table and the two detector heads positioned in each side of the breast. The detector heads can rotate around the breast in order to collect data at several angular orientations for tomographic reconstruction.

\subsection{DAQ system}

In the front-end boards, S8550-01 APD arrays are connected to low noise amplifier and multiplexer ASICs. This ASIC performs the readout of one side of six modules (192 channels), amplification, sampling and storage in analog memories at the system frequency up to $100 \mathrm{MHz}$, as well as the selection of two active channels (192:2 multiplexing) above a common threshold. Analog dataframe composed of 10 samples are digitized in the frontend by 10-bit sampling ADCs, serialized in LVDS bit streams and transmitted to the off-detector system.

The off-detector DAQ system is housed in a $6 \mathrm{U}$ crate with two dedicated buses implemented in CompactPCI backplanes. Two types of electronic boards were developed: the DAQ boards and the TGR and data concentrator board (TGR/DCC board). DAQ boards are responsible for the initial phase of data reduction and pipeline data storage. Parallel algorithmic processing is used in order to minimize dead time while extracting the amplitude and time from the detector pulses. The algorithms are implemented in Xilinx Virtex-II FPGAs with 4 million gates $[5,6]$.

At each TGR the relevant dataframes are transmitted to the acquisition PC where raw data is re-processed for energy and time extraction [6]. From the extracted energy values, the crystal DoI coordinate $z$ is estimated by the energy signal asymmetry between the top and bottom APD channels. For events with more than one active crystal in a detector head, reconstruction position algorithms are used to estimate the first interaction crystal [7].

\section{Detector module performance}

The performance of 24 detector modules was evaluated by detailed measurements. Light yield, energy resolution, DoI resolution and inter-crystal crosstalk were measured in a dedicated setup. The APD-arrays (16 pixels) are polarized with a common bias voltage adjusted for gain $M=50$. The detector signals are processed by discrete charge amplifiers (Cremat CR-101) followed by shaping amplifiers ( $\sim 100 \mathrm{~ns}$ time constant). Peak sensing 12-bit ADCs (CAEN V785) digitize the triggered pulses. One inch $\mathrm{NaI}(\mathrm{Tl})$ scintillator was used in coincidence with the detector module for electronic collimation in some measurements.

\subsection{Relative gain and energy resolution}

The relative detector pixel gain was measured for 768 pixels. The gain dispersion integrates contributions from light yield variation, light collection efficiency, APD quantum efficiency and gain. The r.m.s. relative dispersion of the pixels gain within a detector module was found to be of the order of $10 \%$ on average. The gain dispersion of the detector pixels on the complete scanner is estimated to be $12 \%$ taking into account the contribution from incomplete bias voltage compensation of the relative gain variations of the APD arrays.

The average energy resolution of the $662 \mathrm{keV}{ }^{137} \mathrm{Cs}$ photopeak measured with a flood irradiation was $13 \%$. No significant variations from module to module were found. The APD-array (16 pixels) bias voltage was set for a gain $M=50$.

\subsection{DoI}

The capability to measure the DoI coordinate was assessed in the first 24 detector modules. Light collection asymmetry was calculated and two reference parameters evaluated: (1) slope defined as the top-bottom asymmetry variation/unit length; (2) DoI resolution estimated by the FWHM of asymmetry peak over slope. The asymmetry distributions for events in the $511 \mathrm{keV}$ photopeak in three incident positions along the crystal are shown in Fig. 1. The average value of the slope is $4 \% / \mathrm{mm}$ and the average DoI resolution is about $2.5 \mathrm{~mm}$ (FWHM) not corrected for the $\sim 1 \mathrm{~mm}$ beam width. 


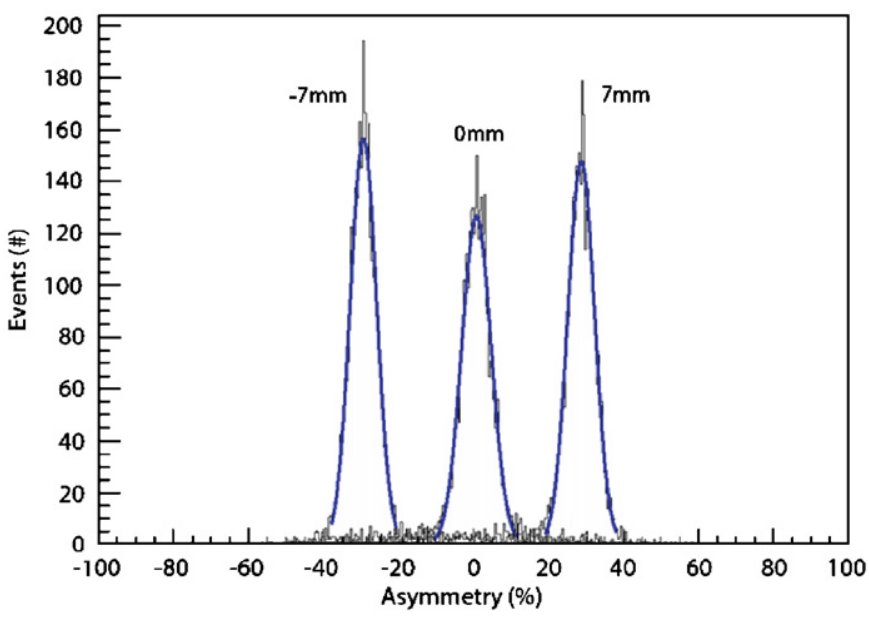

Fig. 1. Distributions of top-bottom APD asymmetry for events in the $511 \mathrm{keV}$ photopeak in three incident positions along the crystal.

\subsection{Inter-pixel cross-talk}

Inter-pixel cross-talk was studied by selecting events with energy deposited in a given crystal and recording the energy distribution in the neighbor channels. As expected, Compton scattered events are predominant in the forward direction. This contribution is reduced as the threshold value in the central crystal is increased. Selecting events near the photopeak of that crystal shows a fraction of instrumental cross-talk of the order of $2.5 \%$.

\section{Clear-PEM performance}

Detailed simulations of the patient, detector and DAQ system are necessary in order to characterize the scanner capabilities, optimize configuration parameters and evaluate its impact on the image quality. For these tasks a simulation framework has been implemented which is partitioned into three modules [8]. The two first modules were developed on the Geant4 Monte Carlo simulation toolkit. The first module allows the description of realistic phantoms and radioisotope decay. The simulation of photon interaction with the scanner is performed in the second module. This partition allows changing detector parameters without requiring the simulation of new emission data. A similar approach was taken for the digitization module which describes the DAQ and TGR system. In Table 1 the baseline parameters that characterize the Clear-PEM used in our simulations are presented.

\subsection{Detection sensitivity}

The expected detection sensitivity to coincidence events was determined. In order to calculate realistic sensitivity values that take into account the influence of the readout system, hits were digitized and the hardware TGR was emulated. Datastream identical to the ones produced by the DAQ system were produced and then fed into the software TGR. The TGR efficiency was studied for
Table 1

Clear-PEM simulation parameters

\begin{tabular}{lll}
\hline & Parameter & Value \\
\hline Crystals and APDs & Light yield and & 27,000 photons $/ \mathrm{MeV}$ \\
& collection efficiency & $30 \%$ \\
& Excess noise factor & 1.75 \\
ASICs & Noise (ENC) & $1000 \mathrm{e}^{-}$ \\
& Nominal gain & $19.3 \mathrm{mV} / \mathrm{fC}$ \\
& Pulse peaking time & $40 \mathrm{~ns}$ \\
& Analog dataframe & $10 \mathrm{samples} @ 50 \mathrm{MHz}$ \\
ADCs & Frequency/resolution & $50 \mathrm{MHz} / 10 \mathrm{bit}$ \\
Off-detector FPGAs & Compton threshold & $35 \mathrm{keV}$ \\
& Event threshold & $125 \mathrm{keV}$ \\
& Compton window & $13 \mathrm{~ns}$ \\
& Coincidence window & $4 \mathrm{~ns}$ \\
Software trigger & Energy window & $350-700 \mathrm{keV}$ \\
\hline
\end{tabular}

Table 2

Count rates for typical exam scenarios

\begin{tabular}{|c|c|c|c|}
\hline Breast size & $\begin{array}{l}\text { Heads separation } \\
(\mathrm{cm})\end{array}$ & $\begin{array}{l}\text { True rate } \\
(\mathrm{kHz})\end{array}$ & Random/true \\
\hline \multirow[t]{2}{*}{ Small } & \multirow[t]{2}{*}{15} & \multirow[t]{2}{*}{2.5} & $44 \%$ (no shield) \\
\hline & & & $28 \%(\mathrm{~Pb}$ shield $)$ \\
\hline \multirow[t]{2}{*}{ Medium } & \multirow[t]{2}{*}{13} & \multirow[t]{2}{*}{11.0} & $25 \%$ (no shield) \\
\hline & & & $20 \%(\mathrm{~Pb}$ shield $)$ \\
\hline \multirow{2}{*}{$\begin{array}{l}\text { Medium with } \\
\text { compression }\end{array}$} & \multirow[t]{2}{*}{10} & \multirow[t]{2}{*}{16.5} & $24 \%$ (no shield) \\
\hline & & & $19 \%(\mathrm{~Pb}$ shield $)$ \\
\hline
\end{tabular}

different coincidence topologies. The fraction of fakes or badly reconstructed events was also analyzed. Fake events are reconstructed with a different topology from the original hit configuration due to some of its information being incorrectly filtered out by the DAQ/TGR system. For a $511 \mathrm{keV}$ point source located in the centre of the FoV the detection sensitivity is $4.3 \%$ with a fake fraction less than $19 \%$. The efficiency for coincidence photoelectric events is about $92 \%$.

\subsection{Estimated count rates}

The presence of activity outside the detector FoV due to normal radiotracer uptake in the torso dominates the system count rates. We use the implementation of the NCAT phantom [9] (128 voxels with $3.25 \mathrm{~mm}^{3}$ resolution) and the detailed detector geometry. For a $10 \mathrm{~cm}$ separation distance the maximum rates per detector head of single events above $5 \mathrm{keV}$ were found to be $2.2 \mathrm{MHz}$. Results on coincidence rates are presented in Table 2 . The true rates were determined for a breast uptake of about $2.1 \mathrm{kBq} / \mathrm{mL}$.

\subsection{Imaging studies}

Imaging studies were performed in order to characterize the Clear-PEM lesion visibility and lesion detection 

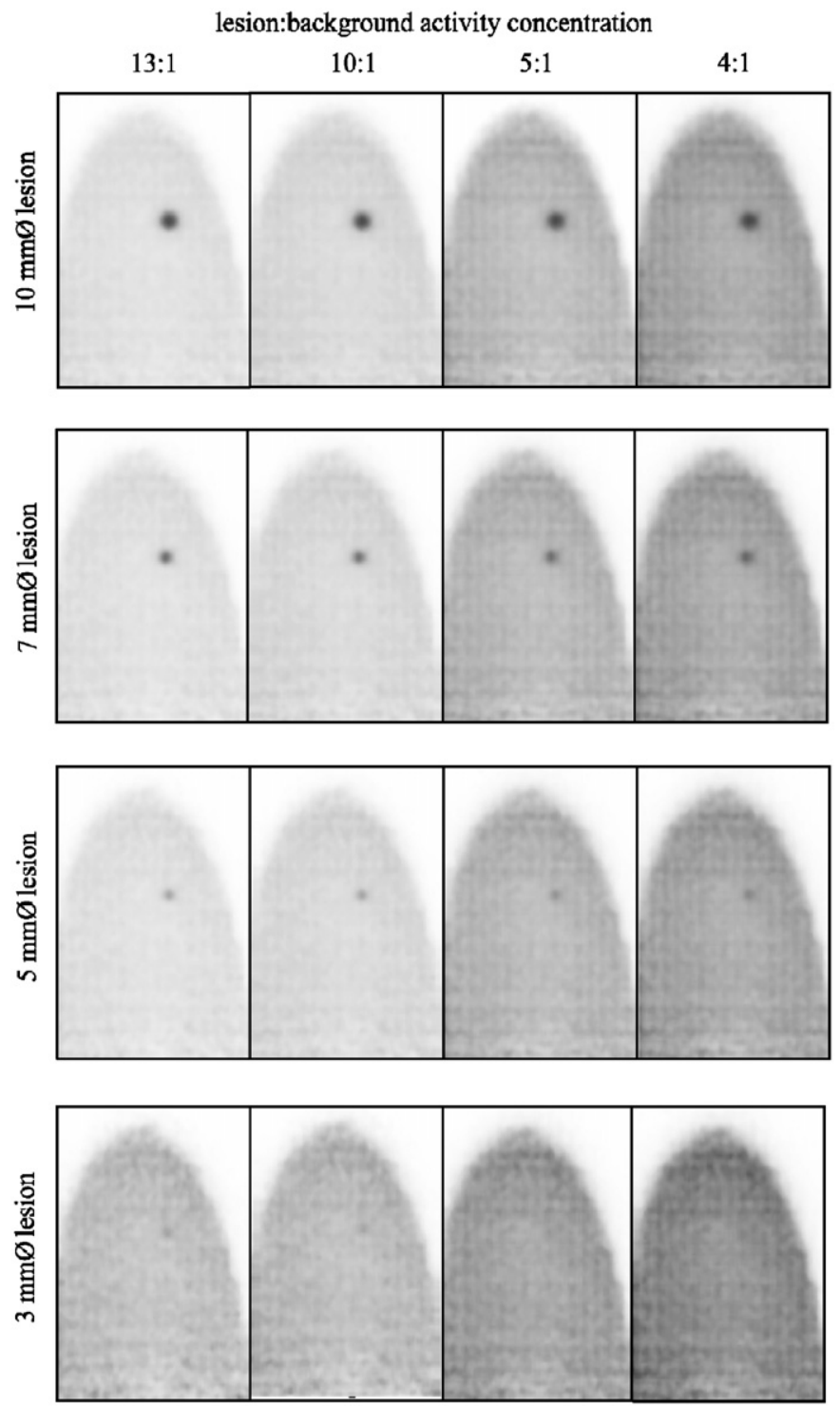

Fig. 2. Representative reconstructed images of the NCAT breast phantom.

significance. Full simulated data using the NCAT breast phantom was reconstructed with 3D-OSEM algorithm in the STIR framework adapted for planar geometries [10].
The simulation considers the full detector model, including gaps and front-end components. The sensitivity map of the Clear-PEM scanner was determined by means of Monte Carlo simulation. Obtained images indicate that the effect of sensitivity variations due to the presence of gaps in the detector geometry can be partially corrected by the full detector sensitivity map.

For lesion visibility assessment, four background activities were considered $(1.6,2.1,3.8$ and $4.8 \mathrm{kBq} / \mathrm{mL})$ and spherical lesions, ranging from 3 to $10 \mathrm{~mm}$ diameters located near the center of the FoV, simulated. Data was obtained for a $5 \mathrm{~min}$ acquisition with two orthogonal projections, $10 \mathrm{~cm}$ separation distance between the detector heads and a 350-700 keV energy window. Reconstructed images are shown in Fig. 2. Although the $3 \mathrm{~mm}$ lesion is still visible for 13:1 lesion-to-background ratio, longer acquisitions may be required in order to achieve a good lesion visibility when in the presence of lower contrast.

\section{Acknowledgments}

This work was supported by the Innovation Agency (AdI) and the Operational Program for Information Society (POSI), Portugal.

\section{References}

[1] C.J. Thompson, et al., IEEE Trans. Nucl. Sci. NS-42 (1995) 1012.

[2] W.W. Moses, Nucl. Instr. and Meth. A 525 (2004) 249.

[3] P. Lecoq, J. Varela, Nucl. Instr. and Meth. A 486 (2002) 1.

[4] M.C. Abreu, IEEE Trans. Nucl. Sci. NS-53 (1) (2006) 71.

[5] E. Albuquerque, et al., The Clear-PEM electronics system, IEEE Trans. Nucl. Sci., to be published.

[6] P. Rodrigues, et al., Performance simulation studies of the ClearPEM DAQ/Trigger system, in: Proceedings of the 14th IEEE-NPSS RT2005, Stockholm, Sweden.

[7] M. Rafecas, et al., Phys. Med. Biol. 48 (2003) 821.

[8] A. Trindade, et al., Breast Cancer Imaging Studies by Monte Carlo Simulation with Clear-PEM, in: Proceedings of MIC/IEEE 2005, Puerto Rico, USA.

[9] W.P. Segars, Development of a new dynamic NURBS-based cardiactorso (NCAT) phantom, Ph.D. Dissertation, The University of North Carolina, USA, 2000.

[10] M.V. Martins et al., Reconstruction of Clear-PEM Data with STIR, in: Proceedings of MIC/IEEE 2005, Puerto Rico, USA. 